\title{
Antiglycation and Antioxidant Activities and HPTLC Analysis of Boswellia sacra Oleogum Resin: The Sacred Frankincense
}

\author{
Ahmed Al-Harrasi $i^{1^{*}}$, Liaqat Ali $^{1}$, Eliezer Ceniviva ${ }^{2}$, Ahmed Al-Rawahi ${ }^{1}$, Javid \\ Hussain ${ }^{1}$, Hidayat Hussain ${ }^{1}$, Najeeb ur Rehman ${ }^{1}$, Ghulam Abbas ${ }^{1,3}$ and Rashid \\ Al-Harrasi ${ }^{1}$ \\ ${ }^{1}$ Department of Biological Sciences and Chemistry, College of Arts and Sciences, University of Nizwa, Birkat Al-Mauz, Nizwa \\ 616, Sultanate of Oman, ${ }^{2}$ CAMAG Laboratory, Sonnenmattstrasse. 11, 4132 Muttenz, Switzerland, ${ }^{3}$ COMSATS Institute of \\ Information Technology, Abbotabad Campus, Abbotabad-22060, Pakistan.
}

*For correspondence: Email: aharrasi@unizwa.edu.om; Tel: +968-92828487; Fax: +968-25446289.

\begin{abstract}
Purpose: To evaluate antiglycation and antioxidant activities as undertake HPTLC analysis of Boswellia sacra resin.

Methods: Sub-fractionation of the crude methanol extract of Hougari regular (HR) grade resin of Boswellia sacra was carried out by vacuum liquid chromatography. Free radical scavenging and antiglycation activities of sub-fractions were characterized in order to assess their anti-aging properties. Furthermore, high performance thin layer chromatography (HPTLC) analysis of Boswellia sacra resins was also carried out.

Results: Polar fractions of the extract obtained exhibited the highest antiglycation activity while nonpolar fractions showed more than 50 \% inhibition in superoxide anion scavenging assay. Scavenging activity of reactive oxygen species results indicate that non-polar sub-fractions showed $>50 \%$ inhibition, except Shabi frankincense (SF) oil which showed $33 \%$ inhibition. Dichloromethane $\left(\mathrm{CH}_{2} \mathrm{Cl}_{2}\right)$ fraction, $40 \%$ dichloromethane $\left(\mathrm{CH}_{2} \mathrm{Cl} 2\right) / n$-hexane sub-fraction, and SF oil showed moderate activity in di(phenyl)-(2,4,6-trinitrophenyl)iminoazanium (DPPH) assay. Furthermore, HPTLC analysis indicates the presence of 11-keto- $\beta$-boswellic acid (KBA) and 3-O-acetyl-11-keto- $\beta$-boswellic acid (AKBA) along with some other terpenoids.

Conclusion: Various sub-fractions of Boswellia sacra exert effective antiglycation and antioxidant activities. The extracts should be studied further for possible formulation into pharmaceutical products.
\end{abstract}

Keywords: Frankincense, Boswellia sacra, Terpenoids, HPTLC, Antioxidant, Antiglycation

Tropical Journal of Pharmaceutical Research is indexed by Science Citation Index (SciSearch), Scopus, International Pharmaceutical Abstract, Chemical Abstracts, Embase, Index Copernicus, EBSCO, African Index Medicus, JournalSeek, Journal Citation Reports/Science Edition, Directory of Open Access Journals (DOAJ), African Journal Online, Bioline International, Open-J-Gate and Pharmacy Abstracts

\section{INTRODUCTION}

Frankincense from Boswellia trees has been used for various therapeutic purposes since the very beginning of the human civilization [1]. It is still used from North Africa to China as a remedy in various formulations for the treatment of inflammation-related disorders, and a multitude of phytochemical and pharmacological properties of the gum resin have been documented [1]. Furthermore, the pharmacological effects of pentacyclic triterpenes, especially those reported from Boswellia species (boswellic acid derivatives) are numerous, and these include anti-inflammatory, hepatoprotective, anti-tumour, anti-HIV, anti-microbial, antifungal, anti-ulcer, 
gastroprotective, hypoglycemic and antihyperlipidemic properties [1]. These boswellic acid derivatives have a similar molecular structure which makes their separation by simple thin-layer chromatography challenging.

High performance thin layer chromatography (HPTLC) is becoming a technique for the routine analysis for the identification of medicinal plants and derived products. The possibility of presenting chromatographic fingerprints as electronic images that can easily be stored, shared and compared for multiple samples in parallel is the principal advantage of the technique over Gas chromatography (GC), highy-performance liquid chromatography (HPLC), and other column chromatography [2,3]. While simplicity and cost efficiency are preserved, separation power and reproducibility as well as traceability of data are significantly improved over classical thin layer chromatography (TLC).

The present study employs HPTLC identification and biological evaluation of Boswellia sacra to explore the antiglycation and antioxidant potentials of the resin derived from Boswellia species.

\section{EXPERIMENTAL}

\section{Chemicals}

Chemicals such as 1,1-diphenyl-2-picrylhydrazyl radical (DPPH), naphthyl-ethylenediamine, sodium nitroprusside, sulfanilic acid, potassium hydrogen phosphate, dipotassium hydrogen phosphate, 3-(2-pyridyl)-5,6-di( $p$-sulfophenyl)1,2,4-triazine, disodium salt (ferrozine), reduced $\beta$-nicotinamide adenine dinucleotide (NADH), 5methylphenazium methyl sulfate (PMS), nitro blue tetrazolium salt (NBT), standard radical scavengers: propyl gallate, 3-t-butyl-4hydroxyanisol, 7,8-dihydroxy flavone; and solvents: dimethylsulfoxide (DMSO), carbon tetrachloride, and ethanol were purchased from Sigma or Fluka and used without further purification. Water used for buffer preparation was deionized using Simplicity water purification system (Millipore).

\section{Plant materials}

The aerial parts of $B$. sacra and the various grades of resin were collected in April and May 2010 from different locations in Dhofar (southern part of Oman) and were supplied by a trusted Dhofari partner (Dr. Saleh Al-Amri). Super hougari green (SHG), hougari regular (HR), royal hougari white (RHW), and hougari yellow $(\mathrm{HY})$ grade resins were collected from Wadi Hougar in Oman, while shabi frankincense (SF) was collected from Wadi Magsyl in Oman. Frankincense Oleogum resins were collected from Boswellia sacra tree by making careful incisions into the bark of the tree without harming the tree. A thick milky-white liquid oozed out and then solidified into pea-sized "tears".

These samples were authenticated by Dr Mustafa Mansi (a taxonomist) of the Department of Biological Sciences and Chemistry, University of Nizwa, Sultanate of Oman and voucher specimen (no. BSHR-01/2012) of the plant was deposited in the herbarium of the Department of Biological Sciences and Chemistry.

\section{Extraction and isolation}

The air-dried ground material (500 g) of HR grade resin was exhaustively extracted with 100 $\%$ methanol at room temperature. The extract was evaporated to yield the residue $(150 \mathrm{~g})$. Subfractionation of the crude methanol extract (150 g) of HR grade resin was carried out by vacuum liquid chromatography. A silica gel column (1000 g, 70-230 mesh, Merck) was used for the fractionation with $\mathrm{CH}_{2} \mathrm{Cl}_{2} / n$-hexane (50:50) as a mobile phase. Various sub-fractions were obtained from liquid chromatography and compiled on the basis of the similarity observed on TLC plates. The essential oils of all available grades of the resin were extracted by hydrodistillation using 8 Quart Stove Still Home Distillation Unit with Clevenger's apparatus until complete exhaustion.

\section{HPTLC analysis}

Chromatography was performed on pre-coated HPTLC silica gel glass plates 60 F254 $(20 \times 10$ cm; E. Merck, Germany) for the development of characteristic fingerprinting profile of the selected samples of the resin from $B$. sacra. Each sample $(0.5 \mathrm{~g})$ was dissolved in HPLC grade methanol (5 $\mathrm{mL}$ ) and sonicated for $10 \mathrm{~min}$. The solution was then centrifuged at $3000 \mathrm{rpm}$ for $10 \mathrm{~min}$ and the upper layer was used for HPTLC analysis after 1:1 dilution with methanol (the standard Boswellia extract was diluted 1:20). Thereafter, 0.5 and $2 \mu \mathrm{L}$ of each sample were applied as bands of $8 \mathrm{~mm}$ using Automatic TLC sampler 4 (CAMAG, Switzerland) with a $25 \mu \mathrm{L}$ syringe. Linear ascending development was carried out in Automatic Developing Chamber (ADC2, CAMAG, Switzerland) saturated with mobile phase for $20 \mathrm{~min}$ at room temperature $\left(24^{\circ} \mathrm{C}\right)$ using a filter paper. For optimum conditions, relative humidity $(\mathrm{RH})$ was controlled at $33 \%$ in ADC2 using a saturated solution of $\mathrm{MgCl}_{2}$. 
Toluene: ethyl acetate: heptane: formic acid $(80: 20: 10: 3, v / v / v / v)$ was used as the mobile phase.

The plates were developed to a distance of $7 \mathrm{~cm}$ from the lower edge of the plate. Drying was carried out for $5 \mathrm{~min}$ in a stream of cold air. For derivatization, the plate was immersed in anisaldehyde-sulphuric acid reagent $(170 \mathrm{~mL}$ of ice-cooled methanol mixed with $20 \mathrm{~mL}$ of acetic acid, $10 \mathrm{~mL}$ of sulfuric acid and $1 \mathrm{~mL}$ of anisaldehyde) followed by heating at $100^{\circ} \mathrm{C}$ for 5 min. Images of the chromatograms were electronically documented with Visualizer (CAMAG Switzerland) under UV $254 \mathrm{~nm}$ before derivatization and under UV $366 \mathrm{~nm}$ and white light after derivatization. The identity of the zones in the sample corresponding to 3-O-acetyl-11keto- $\beta$-boswellic acid (AKBA) was confirmed by comparing their densitometrically-obtained UV spectra with those of a AKBA reference standard (Phytolab, Vestenbergsgreut, Germany). HPTLC according to PhEur 7 Monograph for Indian Frankincense. Mobile phase: Anhydrous formic acid-heptane-ethyl acetate-toluene $(3+10+$ $20+80, \mathrm{v} / \mathrm{v} / \mathrm{v} / \mathrm{v})$. Detection: UV light at $254 \mathrm{~nm}$. Reference compound: KBA $=11$-keto- $\beta$ boswellic acid.

Cytotoxicity and antiradical studies against the 1,1-diphenyl-2-picrylhydrazyl radicals were carried out at $37^{\circ} \mathrm{C}$, while the enzymatic reactions and superoxide scavenging studies were carried out at $28{ }^{\circ} \mathrm{C}$. All studies were performed in 96 -well microtitre plates using SpectraMax-340 and SpectraMax-384 spectrophotometers (Molecular Devices, CA, USA).

\section{Antiglycation assay}

Bovine serum albumin (BSA, $10 \mathrm{mg} / \mathrm{mL}$ ) was dissolved in $67 \mathrm{mM}$ phosphate buffer $(20 \mu \mathrm{L})$ of $\mathrm{pH} 7.4$ which incorporated $50 \mathrm{mg} / \mathrm{mL}$ anhydrous glucose $(20 \mu \mathrm{L})$. Thereafter, a $3 \mathrm{mM}$ sodium azide $(20 \mu \mathrm{L})$ was added to inhibit bacterial growth. For assessment of antiglycation activity, each fraction $(20 \mu \mathrm{L} ; 1 \mathrm{mg} / 1000 \mu \mathrm{L})$ was mixed and the mixture (60 $\mu \mathrm{L}$ in each well of 96-well plate) was incubated for a week at $37{ }^{\circ} \mathrm{C}$. A blank sample containing only BSA dissolved in phosphate buffer and positive control sample containing both BSA and glucose, were prepared and incubated for a week at $37{ }^{\circ} \mathrm{C}$. After incubation in 96- well plate for a week, samples were removed and cooled to room temperature, amd $6 \mu \mathrm{L}$ of $100 \%$ trichloroacetic acid (TCA) was added to each well. The supernatant containing unbound glucose, inhibitor and test sample were removed after centrifugation at 14,000 rpm for 4 min, and $60 \mu \mathrm{L}$ of PBS ( $\mathrm{pH} \mathrm{10)} \mathrm{was} \mathrm{added} \mathrm{to}$ dissolve the pellets left. Comparison of fluorescence intensity at $370 \mathrm{~nm}$ excitation and emission at $440 \mathrm{~nm}$ was obtained by spectrofluorimetry (RF-1500, Shimadzu, Kyoto, Japan) [4]. Rutin was used as standard inhibitor. Inhibition (\%) was calculated as in Eq 1

Inhibition $=100-\{($ As $/ A b) \times 100\}$

where $A s$ and $A b$ are the absorbance of sample and blank, respectively.

\section{Superoxide anion scavenging assay}

Superoxide scavenging activities of the samples were determined by the method described by Gaulejac et al [5] with some modifications. The reaction was performed in triplicate in a 96-well plate and absorbance was measured on a multiplate reader (SpectraMax 340, Molecular Devices, CA, USA). The reaction mixture contained $40 \mu \mathrm{L}$ of nicotinamide adenine dinucleotide (NADH), $40 \mu \mathrm{L}$ of nitroblue tetrazolium (NBT), $90 \mu \mathrm{L}$ of $0.1 \mathrm{M}$ phosphate buffer (pH 7.4) and $10 \mu \mathrm{L}$ of the test compound pre-read at 560 $\mathrm{nm}$. The reaction was initiated by the addition of $20 \mu \mathrm{L}$ of phenazine methosulphate (PMS), and incubated at room temperature for 5 min. Formation of blue-colored formazan dye was measured at $560 \mathrm{~nm}$. Control contained 10 $\mu \mathrm{L}$ of dimethyl sulfoxide (DMSO), instead of the test samples. The solutions of NBT, NADH and PMS were prepared in phosphate buffer, while the test samples were dissolved in DMSO.

\section{DPPH free radical scavenging assay}

Free radical scavenging activity of the test samples were determined by measuring the change in absorbance of DPPH (I,I-Diphenyl-2-picrylhydrazyl radical) spectrophotometrically at $515 \mathrm{~nm}$ [6]. The reaction mixture comprised of $95 \mu \mathrm{L}$ of ethanol solution of $\mathrm{DPPH}$ and $5 \mu \mathrm{L}$ of the test sample dissolved in DMSO. Total reaction volume was 100 $\mu \mathrm{L}$, with final concentrations of $300 \mu \mathrm{M}$ and 1000 $\mu \mathrm{M}$ of DPPH and test compound, respectively. The reaction mixture was then incubated at $37^{\circ} \mathrm{C}$ for $30 \mathrm{~min}$. After incubation, decrease in absorption was measured at $515 \mathrm{~nm}$ spectrophotometrically (Molecular Devices, CA, USA). The control contained $5 \mu \mathrm{L}$ of DMSO instead of the test sample and the reactions were performed in triplicates. To avoid solvent evaporation during incubation, the 96-well plate was covered with parafilm immediately after the addition of DPPH solution and the reaction mixtures were thoroughly mixed by shaking the plate for $1 \mathrm{~min}$. The absorbance of the yellow-colored reduced form of DPPH produced after incubation was measured at $562 \mathrm{~nm}$ using a multiplate reader (SpectraMax- 
340 ), and radical scavenging activity (RSA, \%) was determined according to Eq 2.

$\% \operatorname{RSA}=100-\{\mathrm{At} / \mathrm{Ac}) \times 100\}$

where, At is the absorbance of radicals and formazan dye in the presence of the test sample and $\mathrm{Ac}$ is the absorbance of control, formazan dye without test sample.

\section{Statistical analysis}

The data obtained were analyzed statistically using Statistic Analysis System (SAS, version 9.1). Each experiment was repeated three times and values expressed are means \pm standard error. Differences were considered significant at $p<0.05$.

\section{RESULTS}

\section{HPTLC fingerprinting profile}

Boswellic acids are pharmacologically active compounds isolated from the resins (frankincense) of various species of Boswellia. HPTLC allows rapid investigation, identification and comparison of the quality of different kinds of frankincense (Olibanum). In the present study, the HPTLC profile of the crude resin (Figure 1; tracks 3 and 4 ), the crystalline medium polarity fraction (tracks 5 and 6), and the hydrodistillate essential oil (tracks 7 and 8 ) were analyzed at two quantitative levels $(0.5$ and $2.0 \mu \mathrm{L})$. Boswellia serrata extract (track 1) and the gum resin (track 2), and compared with the standard sample, 3-O-acetyl-11-keto- $\beta$-boswellic acid (AKBA, track 9). All samples showed the presence of 11-keto- $\beta$-boswellic acid (KBA, lower arrow, track 8) and 3-O-acetyl-11-keto- $\beta$ boswellic acid (AKBA, upper arrow, track 8) with different concentration (intensity) in all the three test samples (Figure 1). The same compounds were identified in approximately the same concentration for the standard $B$. serrata extract and the gum resin (KBA and AKBA, black arrows, tracks 1 and 2). These results were further confirmed by matching the UV spectra of the corresponding bands in the test samples with those of the standard AKBA.

These results and comparison with the literature [7] confirm the identity of the investigated samples as $B$. sacra based on various concentrations of KBA and AKBA. The concentrations of the same compounds in $B$. serrata were approximately equal. These observations were further confirmed through derivatization of the HPTLC plate with the anisaldehyde reagent (Figure 2).

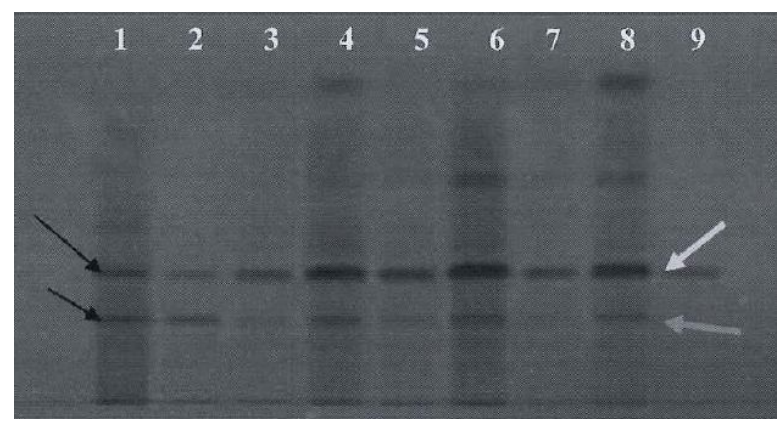

Figure 1: Detection and comparison of boswellic acids in different samples of Boswellia sacra. Track 1 = Boswellia serrata extract (CAMAG); Track 2 = Boswellia serrata gum (CAMAG); Track 3 \& 4 = Boswellia sacra resin (test sample, 0.5 and $2.0 \mu \mathrm{L}$, respectively); Track 5 \& 6 Boswellia sacra crystalline fraction (test sample, 0.5 and $2.0 \mu \mathrm{L}$ respectively); Track $\mathbf{7} \& \mathbf{8}=$ Boswellia sacra resin (test sample, 0.5 and $2.0 \mu \mathrm{L}$ respectively); Track 9 = standard AKBA

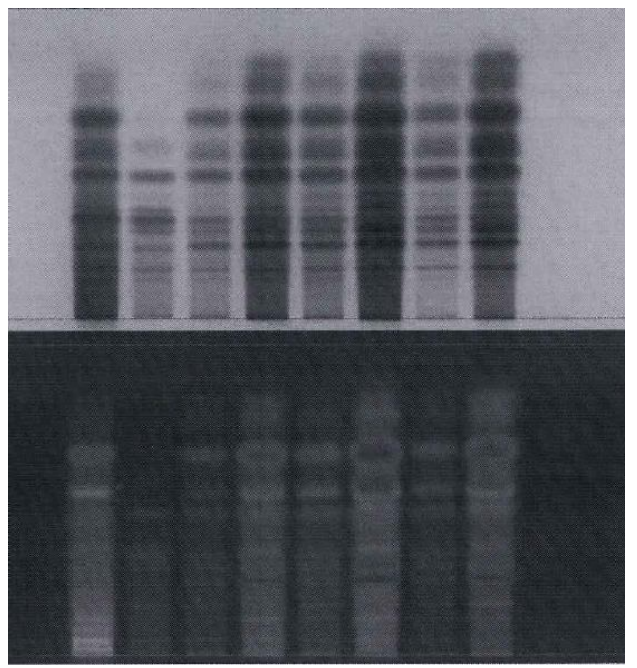

Figure 2. Detection and comparison of boswellic acids in different samples of Boswellia sacra. Track 1 = Boswellia serrata extract (CAMAG); Track 2 = Boswellia serrata gum (CAMAG); Track 3 \& 4 = Boswellia sacra resin (test sample, 0.5 and $2.0 \mu \mathrm{L}$, respectively); Track 5 \& 6 Boswellia sacra crystalline fraction (test sample, 0.5 and $2.0 \mu \mathrm{L}$ respectively); $\operatorname{Track} \mathbf{7} \& \mathbf{8}=$ Boswellia sacra resin (test sample, 0.5 and $2.0 \mu \mathrm{L}$ respectively); Track $9=$ standard AKBA.

\section{Antiglycation activity}

All the tested samples showed varied inhibitory potential in vitro at a concentration of $1 \mathrm{mg} / 1000$ $\mu \mathrm{L}$ (Table 1). The sub-fraction obtained with 36 $\% \quad \mathrm{CH}_{2} \mathrm{Cl}_{2} / n$-hexane showed the highest inhibitory activity (69.5\%), followed by $2 \%$ methanol/ $\mathrm{CH}_{2} \mathrm{Cl}_{2}(66.9 \%)$ and Royal lower (RL) oil $(54.3 \%)$. The sub-fraction obtained with $40 \%$ methanol/ $\mathrm{CH}_{2} \mathrm{Cl}_{2}$ and Shabi frankincense (SF) oil showed moderate activity in antiglycation assay 
Table 1: Antiglycation and antioxidant activities (mean \pm standard error) of various fractions of Boswellia serrata

\begin{tabular}{lccc}
\hline \multirow{2}{*}{ Sample } & \multicolumn{3}{c}{ Inhibitory activity (\%) } \\
\cline { 2 - 4 } & Antiglycation & DPPH & Superoxide \\
\hline $\mathrm{HR}$ oil & a & $16.30 \pm 0.04$ & $56.40 \pm 0.01$ \\
$\mathrm{RL}$ oil & $54.30 \pm 0.02$ & $5.60 \pm 0.02$ & $50.50 \pm 0.02$ \\
$\mathrm{RU}$ oil & $5.30 \pm 0.06$ & $2.80 \pm 0.01$ & $52.80 \pm 0.05$ \\
$\mathrm{SF}$ oil & $37.20 \pm 0.01$ & $33.40 \pm 0.04$ & $33.10 \pm 0.08$ \\
Crude extract & $10.90 \pm 0.03$ & $14.30 \pm 0.05$ & $49.00 \pm 0.02$ \\
Pure n-hexane & $6.80 \pm 0.02$ & $6.60 \pm 0.03$ & $50.80 \pm 0.14$ \\
$36 \% \mathrm{CH}_{2} \mathrm{Cl}_{2} / n$-hexane & $69.50 \pm 0.06$ & $11.90 \pm 0.12$ & $13.40 \pm 0.03$ \\
$40 \% \mathrm{CH}_{2} \mathrm{Cl}_{2} / n$-hexane & $14.40 \pm 0.01$ & $31.30 \pm 0.03$ & $16.90 \pm 0.05$ \\
$60 \% \mathrm{CH}_{2} \mathrm{Cl}_{2} / n$-hexane & $7.80 \pm 0.01$ & $15.60 \pm 0.08$ & $23.40 \pm 0.01$ \\
$80 \% \mathrm{CH}_{2} \mathrm{Cl}_{2} / n$-hexane & $10.30 \pm 0.03$ & $13.30 \pm 0.05$ & $33.50 \pm 0.03$ \\
$\mathrm{Pure} \mathrm{CH} \mathrm{Cl}_{2}$ & $12.40 \pm 0.07$ & $31.80 \pm 0.14$ & $49.70 \pm 0.04$ \\
$2 \% \mathrm{MeOH}_{\mathrm{CH}} \mathrm{Cl}_{2}$ & $66.90 \pm 0.02$ & $10.90 \pm 0.08$ & $6.20 \pm 0.01$ \\
$8 \% \mathrm{MeOH} / \mathrm{CH}_{2} \mathrm{Cl}_{2}$ & $9.90 \pm 0.03$ & $12.10 \pm 0.07$ & $11.80 \pm 0.02$ \\
$40 \% \mathrm{MeOH} / \mathrm{CH}_{2} \mathrm{Cl}_{2}$ & $41.20 \pm 0.04$ & $10.00 \pm 0.2$ & $50.80 \pm 0.04$ \\
Standard & Rutin & Propyl gallate & Propyl gallate \\
& $(82.50 \%)$ & $(90.30 \%)$ & $(92.50 \%)$ \\
\hline
\end{tabular}

${ }^{a} H R=$ Hougari regular; $R L=$ Royal lower; $R U=$ Royal upper; $S F=$ Shabi frankincense

whereas the other samples were weakly active or completely inactive (Table 1).

\section{Superoxide anion scavenging activity}

The results obtained indicate that the non-polar sub-fractions exhibited $>50 \%$ inhibition, except n-hexane fraction, $\mathrm{CH}_{2} \mathrm{Cl}_{2}$ fraction and $40 \%$ methanol/ $\mathrm{CH}_{2} \mathrm{Cl}_{2}$ fraction all of which exhibited approximately the level same inhibition which was close to $50 \%$. Other test samples showed <

\section{DISCUSSION}

Triterpenic acids are an important group of natural compounds with confirmed pharmacological activity [1]. They occur in many medicinal herbs and plants. The similarity of their chemical structures makes their TLC separation very difficult. There are some chromatographic systems described in the literature [8-11], but they do not offer better separation, and the result is poor overall yield of the pure natural products. In this regard, our approach using HPTLC offers more effective separation and higher yield.

Oxidation process plays an important role in glycation end-products (AGEs) formation and is essential to many living organisms for the production of energy to fuel the biological processes. On the other hand, reactive oxygen species (ROS) can damage DNA and thus cause mutation and chromosomal damage [12-14]. Furthermore, production of excessive free radicals stimulates oxidative damage which is responsible for more than one hundred disorders
$50 \%$ inhibition was close to $50 \%$. Other test samples showed $<50 \%$ inhibition.

\section{DPPH free radical scavenging activity}

$\mathrm{CH}_{2} \mathrm{Cl}_{2}$ fraction and the sub-fraction of $40 \%$ $\mathrm{CH}_{2} \mathrm{Cl}_{2} / n$-hexane fraction, as well as SF oil showed moderate activity (Table 1 ), while the other samples were largely inactive. Standard rutin showed $82.5 \%$ inhibition of glycation at 3 $\mathrm{mM}$ concentration with $\mathrm{IC}_{50}$ of $98.01 \pm 2.03 \mu \mathrm{M}$. in humans including atherosclerosis, coronary heart disease, neurodegenerative disorders, cancer, and aging process. Therefore, agents with antioxidative or metal-chelating properties may retard the process of AGEs formation by preventing metal-catalyzed glucose oxidation [12-14].

Free radicals, such as hydroxyl radical, are generated from sequential reduction of oxygen during the normal course of aerobic metabolism. Over-abundant radicals cause oxidative stress which can lead to cell injury and tissue damage [15]. B. sacra extract is a potential source of natural antioxidants, and incorporation of these extract into foods could enhance their nutritional and antioxidant potentials.

DPPH radicals are widely used to investigate the scavenging activity of natural compounds. These free radicals are stable in ethanol and show maximum absorbance at $517 \mathrm{~nm}$. When DPPH radicals encounter a proton-donating substance such as an antioxidant, the radicals are scavenged and their absorbance reduced [15]. $B$. sacra extracts and essential oils showed 
scavenging activities against DPPH radicals. This is not surprising since they contain a large variety of terpenes [16], which could be electron donors, and hence can react with free radicals to convert them to more stable products and terminate radical chain reaction.

The results gleaned from antioxidant and antiglycation assays indicated that the fractions obtained by the use of a $n$-hexane or $\mathrm{CH}_{2} \mathrm{Cl}_{2}$ solvent were comparatively more active in almost all the assays. Furthermore the crystalline subfractions and the more polar fractions (40\% methanol $/ \mathrm{CH}_{2} \mathrm{Cl}_{2}$ ) also showed comparatively higher activity than the other tested samples. These observations indicate that overall activity increases the purer the sample and the higher the polarity of the fraction. The polar subfractions obtained when methanol solvent system was added contain chemical compounds that are believed to be responsible for their higher antiglycation activity. Our assumption is supported by a study on Plantago asiatica which showed higher antiglycation activity (75 \%) for polar fractions [17].

\section{CONCLUSION}

An HPTLC method has been applied successfully for the simultaneous fingerprint identification of boswellic acid derivatives in $B$. sacra.

The method was found to be simple and specific and suitable for further qualitative analysis of the plant material. The tested materials possessed high antioxidant and antiglycation activities. Thus the plant may offer additional sources of ingredients that can be formulated into products for health promotion.

\section{ACKNOWLEDGMENT}

The authors would like to thank The Oman Research Council (TRC) for generous support through the funding of a project (ref no. ORG/CBS/10/002) and the University of Nizwa via funding of another project (ref no. A109-10UON/28/A \& S/IF).

\section{REFERENCES}

1. Shah BA, Qazi GN, Taneja SC. Boswellic acids: a group of medicinally important compounds. Nat Prod Rep 2009; 26: 72-89.
2. Cert A, Moreda W, Perez-Camino MC. Chromatographic analysis of minor constituents in vegetable oils. $J$ Chromatogr A 2000; 881: 131-148

3. Bazylak G, Brózik H, Sabanty W. Combined SPE and HPTLC as a screening assay of urinary cotinine from male adolescents exposed to environmental tobacco smoke. Pol J Environ Stud 2000; 9: 113123.

4. Matsuda $H$, Wang $T$, Managi $H$, Yoshikawa $M$. Structural requirements of flavonoids for inhibition of protein glycation and radical scavenging activities. Bioorg Med Chem 2003; 11: $5317-5323$

5. Gaulejac NSC, Glories $Y$, Vivas $N$. Free radical scavenging effect of anthocyanins in red wines. Food Res Int 1999; 32: 327-333

6. Lee SK, Mbwambo ZH, Chung $H$, Luyengi L, Gamez EJ, Mehta RG, Kinghorn D, Pezzuto JM. Evaluation of the antioxidant potential of natural products. Comb Chem High Throughput Screen 1998; 1: 35-46

7. Meier B, Spriano D. Modern HPTLC-A pefect tool for quality control of herbals and their preparations. $J$ AOAC Int 2010; 93: 1399-1409

8. Banerjee $A$, Sane $R T$, Mangaonkar $K$, Shailajan $S$, Deshpande A, Gundi G. Quantitation of oleanolic acid in Oldenlandia corymbosa L. wholeplant powder by high-performance thin-layer chromatography. J Planar Chromatogr 2006; 19: 68-72

9. Baricevic $D$, Sosa $S$, Della $L R$, Tubaro A, Simonovska $B$, Krasna A, Zupancic A. Topical anti-inflammatory activity of Salvia officinalis $L$. leaves: The relevance of ursolic acid. J Ethnopharmacol 2001; 75: 125132

10. Wagner $H$, Bladt $S$ (1996) Plant Drug Analysis, Springer-Verlag, Berlin/ Heidelberg/New York, pp 305-326

11. Shetty $P$, Mangaonkar $K$, Sane RT. HPTLC determination of ursolic acid in Alstonia scholaris. $J$ Planar Chromatogr 2007; 20: 65-68

12. Jothy SL, Zuraini Z, Sasidharan S. Phytochemicals screening, $D P P H$ free radical scavenging and xanthine oxidase inhibitiory activities of Cassia fistula seeds extract. J Med Plants Res 2011; 5: 1941-1947

13. Pong K. Oxidative stress in neurodegenerative disease: Therapeutic implication or superoxide dismutase mimetics. Expert Opin Biol Ther 2003; 3: 127-139

14. Sandhya B, Manoharan S, Lavanya GS, Manmohan CR. Lipid peroxidation and antioxidant status in prostate cancer patients. Indian J Sci Technol 2010; 3: 83-86

15. Amadou I, Le GW, Shi YH. Effect of Boiling on the Cytotoxic and Antioxidant Properties of Aqueous Fruit Extract of Desert Date, Balanites aegyptiaca (L) Delile. Tropical J Pharm Res 2012; 13: 437444.

16. Al-Harrasi A, Al-Saidi S. Phytochemical Analysis of the Essential Oil from Botanically Certified Oleogum Resin of Boswellia sacra (Omani Luban). Molecules 2008; 13: 2181-2189.

17. Choi SY, Jung SH, Lee HS, Park KW, Yun BS, Lee KW. Glycation inhibitory activity and the identification of an active compound in Plantago asiatica extract. Phytother Res 2008; 22: 323-329. 